\title{
RESPONSE OF WILD MAMMALS TO SEASONAL SHRINKING-AND-EXPANSION OF HABITATS DUE TO FLOODING REGIME OF THE PANTANAL, BRAZIL
}

\author{
MAMEDE, S. B. and ALHO, C. J. R. \\ Universidade para o Desenvolvimento do Estado e da Região do Pantanal - UNIDERP, \\ Rua Ceará, 333, CEP 79003-010, Campo Grande, MS, Brazil \\ Correspondence to: Cleber José Rodrigues Alho, Universidade para o Desenvolvimento do Estado e da Região do Pantanal \\ - UNIDERP, Rua Ceará, 333, CEP 79003-010, Campo Grande, MS, Brazil, e-mail: alho@ unb.br \\ Received October 26, 2004 - Accepted January 14, 2006 - Distributed November 1, 2006
}

(With 1 figure)

\begin{abstract}
The Pantanal is a large savanna wetland $\left(138,183 \mathrm{~km}^{2}\right.$ in Brazil), important for its wildlife, fed by tributaries of the upper Paraguay River, center of South America (Brazil, touching Bolivia and Paraguay). Uplands are plateaus $\left(250-1,200 \mathrm{~m}\right.$ high, $215,000 \mathrm{~km}^{2}$ in Brazil) and flatland is the Pantanal $(80-150 \mathrm{~m}$ high, $147,574 \mathrm{~km}^{2}$ in Brazil). Rivers are slow moving when they meet the flatland (slope $0.3-0.5 \mathrm{~m} / \mathrm{km}$ east-west; $0.03-0.15 \mathrm{~m} / \mathrm{km}$ north-south), periodically overflowing their banks, creating a complex seasonal habitat range. Recurrent shallow flooding occupies $80 \%$ of the Pantanal; during the dry season flooded areas dry up. Fluctuating water levels, nutrients and wildlife form a dynamic ecosystem. A flooding regime forms distinct sub-regions within the Pantanal. A mammal survey was carried out in the sub-region of the Rio Negro from April, 2003 through March, 2004 to study the diversity and abundance of terrestrial mammals during the dry and flooding seasons. A total of 36 species were observed in the field. The capybara Hydrochaeris hydrochaeris was the most frequent species, followed by the crab-eating-fox Cerdocyon thous and the marsh deer Blastocerus dichotomus. The highest abundance of species was observed during the dry season (August and September), when there is a considerable expansion of terrestrial habitats, mainly seasonally flooded grassland. Animal abundance (in terms of observed individual frequencies) varied during the dry and wet seasons and the seasonally flooded grassland was the most utilized habitat by mammals in the dry season.
\end{abstract}

Keywords: habitats, mammals, pantanal, seasonality, wetland.

\section{RESUMO}

\section{Resposta de mamíferos silvestres do Pantanal ao encolhimento-e-expansão sazonal de habitats devido ao regime de enchente}

O Pantanal é uma área inundável (138.183 km² no Brasil), importante globalmente por sua biodiversidade, alimentado por tributários da margem esquerda do alto Rio Paraguai, no centro da América do Sul (Brasil, tocando a Bolívia e o Paraguai). As terras altas do entorno são o planalto (250-1200 m de altitude, $215.000 \mathrm{~km}^{2}$ no Brasil), e a planície é o Pantanal (80-150 m de altitude, $147.574 \mathrm{~km}^{2}$ no Brasil). Os rios movem-se lentamente quando encontram a planície (declividade de 0,3-0,5 m/km leste-oeste; 0,03-0,15 m/km nortesul), periodicamente transbordando suas margens, criando um complexo de habitats sazonais. Inundação rasa e recorrente ocupa $80 \%$ do Pantanal; áreas inundáveis secam na estação seca. Flutuação do nível da água, nutrientes e espécies silvestres formam um ecossistema dinâmico. Diferentes tipos de inundação formam diferentes tipos de pantanais. Um censo de mamíferos silvestres foi feito no Pantanal do Rio Negro, de abril de 2003 até março de 2004, para estudar a diversidade e a abundância de mamíferos terrestres durante as estações seca e cheia. Um total de 36 espécies de mamíferos foi observado no campo. A capivara Hydrochaeris hydrochaeris foi a espécie mais freqüente, seguida do lobinho Cerdocyon thous e do cervo-do-Pantanal Blastocerus dichotomus. A maior freqüência de espécies de mamíferos observada 
(abundância) foi registrada em agosto e setembro, coincidindo com a época de maior expansão de habitats sazonalmente inundáveis. A abundância de animais (em termos de freqüência de indivíduos observados) varia entre a estação seca e cheia, sendo que os campos sazonalmente inundáveis são os habitats mais utilizados pelos animais na estação seca.

Palavras-chave: áreas inundáveis, habitats, mamíferos, pantanal, sazonalidade.

\section{INTRODUCTION}

The Pantanal is a savanna wetland recognized as one of the most important freshwater systems in the world for its wildlife (Alho et al., 1988; Alho et al. 2003; Alho, 2005; Alho \& Gonçalves, 2005; PCBAP, 1997). It is fed by tributaries of the upper Paraguay River. These major rivers feeding the Pantanal are (from north to south): the Paraguay, Jauru, Cabaçal, Sepotuba, Bento Gomes, Cuiabá, São Lourenço, Correntes, Itiquira, Piquiri, Taquari, Negro, Aquidauana, Miranda, Nabileque and Apa (ANA, 2004). The Pantanal, as a floodplain, retains the water that flows down from the plateaus where the rivers upstream are located.

The altitude in the floodplain varies from 80 to $150 \mathrm{~m}$ above sea level and the topographical gradients are weak, with the slope ranging from 0.3 to $0.5 \mathrm{~m} / \mathrm{km}$ east-west and 0.03 to $0.15 \mathrm{~m} / \mathrm{km}$ north-south (Franco \& Pinheiro, 1982; Alho, 2005). The river slope in the highlands is about $0.6 \mathrm{~m} / \mathrm{km}$, while on the plain it is 0.1 to $0.3 \mathrm{~m} / \mathrm{km}$. A digital model for treatment of topographic maps of the sub-region of Nhecolândia has shown that in altitudes lower than $100 \mathrm{~m}$, the floodplain is flatter, while in altitudes above $100 \mathrm{~m}$ there is more slope in the terrain, going east, in the direction of the Maracaju uplands (Fernandes et al., 1996). When the floodplain reaches the plateau, the altitude suddenly changes to $150-400 \mathrm{~m}$. This low slope on the plain is a hindrance to drainage of the tributaries on the left margin of the Paraguay River.

The rainy season, in which most rain falls between November and March having differences between the northern and the southern regions, varies annually from 1,200 to $1,300 \mathrm{~mm}$ across the region and in some years could reach $2,000 \mathrm{~mm}$. Rainfall is more intensive in the northern uplands than in the south. In the Pantanal of the Negro River, the maximum precipitation occurs in December (near $300 \mathrm{~mm}$ ) and the minimum occurs in June (near $20 \mathrm{~mm}$ ).

A study scanning multichannel microwave radiometers to reveal inundation patterns in the Pantanal showed maximum inundation occurring as early as February in the northern sub-regions and as late as June in the south, as a result of the delayed drainage of the region (Hamilton et al., 1996). An area of $131,000 \mathrm{~km}^{2}$ was inundated annually during nine years of observation, between 1979 and 1987. An average area of $53,000 \mathrm{~km}^{2}$ is inundated annually and monthly estimates of the total area inundated range from 11,000 to $110,000 \mathrm{~km}^{2}$.

The distinct annual flow of the rivers, causing wet and dry seasons, results in hydrological seasonality with productivity of feeding and reproductive grounds for wildlife due to biochemical cycles (Junk, 1993; Vinson \& Hawkins, 1998; Wantzen \& Junk, 2000; Alho \& Gonçalves, 2005). The habitats change due to the water discharge carrying nutrients and sediments, depositing inorganic and organic matters that enrich the microhabitats, favoring the proliferation of microorganisms, invertebrates, fishes, amphibians, reptiles, birds and mammals.

As a wetland ecosystem, the Pantanal is characterized by an indistinct and ever-changing boundary between water and land. A variety of habitats is present, offering clear seasonality in food production and other ecological resources, and therefore a great number of animals can thrive with minimal competition. The ever-changing patterns of floating vegetation, annual plants, solid ground and open water provide ample niches for a wide range of plants and animals (Alho et al., 2003; Alho \& Gonçalves, 2004; Alho, 2005). Flooding is the most important ecological phenomenon in the Pantanal. Every year many parts of the Pantanal change from terrestrial into aquatic habitats.

The degree of inundation creates a range of major habitats. The rivers are lined with gallery forests and there are dense semideciduous forests in the higher areas. The remainder of the habitats is either grassland or seasonally flooded grassland. There are 3,400 higher plant species in the upper Paraguay basin, 1,863 phanerogamic species in the Pantanal (ANA, 2004; Alho \& Gonçalves, 2005). In terms of flora and fauna, the region is 
related to the Cerrado biome, and there is a strong Amazonian influence as well. The mix of major vegetation formations that occurs in the Pantanal, plus the presence of local endemism, has resulted in diverse and abundant fauna. Many endangered species can be observed in the Pantanal (Alho et al., 2003). Caimans Caiman crocodilus are widespread and capybaras Hydrochaeris hydrochaeris are common throughout the Pantanal. Waterfowl are exceptionally abundant, including herons, egrets, storks, ibises, spoonbills and kingfishers. There are 263 fish species, 400 when the plateaus are included; 179 reptile species, 85 in the Pantanal and 94 on the plateaus; 80 species of amphibians, 35 in the Pantanal and 45 on the plateaus; 661 birds, 444 in the Pantanal; and 195 mammals (Alho et al., 2003; Alho \& Gonçalves, 2005; ANA, 2004). However, threats such as fire, deforestation and conversion of natural habitats into crop fields and pasture, water pollution and other factors have an impact on the natural habitats and their associated fauna.

The purpose of this work is to show the influence of the annual hydrological flow of the rivers of the Pantanal on the dynamics and maintenance of the natural habitats supporting wild mammals. We indicate that in different habitats the abundance of some species of mammals vary according to the available seasonal habitat. A second goal is to emphasize the importance of forested and arboreal habitats (gallery and semideciduous forests, patches of cerradão and cerrado) to mammal species of the Pantanal, mainly during the flooding period.

\section{STUDY AREA}

The study area is part of the sub-region of the Negro River, where the field station of UNIDERP (Instituto de Pesquisa do Pantanal - IPPAN) is located: $30^{\circ} 18^{\prime \prime} \mathrm{S}$ and $\left.55^{\circ} 18^{\prime} \mathrm{W}\right)$. The IPPAN and neighboring ranches (fazendas Santa Maria, Chão Parado, Campo Lourdes, Cordilheira, São Geraldo, Santa Marta, Conquista, Bocaiúva, Proteção, Olhos D’Água, Santana, Serro Azul, Serro Alto, Nova Espanha, Taboco and São Roque) were intensively surveyed for mammals. Five linear but not strait transects were established to survey the animals: Transect A with $2 \mathrm{~km}$ of extension; Transect B, $2 \mathrm{~km}$; Transect C, $30 \mathrm{~km}$; Transect D, $4.6 \mathrm{~km}$; Transect E, $2.5 \mathrm{~km}$. An area of approximately 4,000 hectares was surveyed.
The Pantanal of the Negro River is limited in the north by the alluvial fans of the Taquari River and in the south by the Miranda and Aquidauana Rivers. The flooding period is from March to May but sometimes it may last up to six months (TNC, 2003). The slope is low, $20 \mathrm{~cm} / \mathrm{km}$. The dominant vegetation formation is Cerrado with the occurrence of gallery and mesophytic forests and the presence of seasonally flooded fields connected with depressions forming ponds (PCBAP, 1997; ANA, 2004).

\section{METHODS}

Five linear but sinuous transects (sizes varying from $2 \mathrm{~km}$ to $30 \mathrm{~km}$ ) crossing all kinds of habitats were established to survey the animals. A digital map and a Landsat 7 image of the region were used to set the transects. A Garmin 12 XL GPS was used to locate the animals. The animal censuses were carried out over 11 months (from April, 2003 through March, 2004, except July) surveying throughout the transects by car, walking, on horse-back and by boat (along the Correntoso River), looking for direct sightings of mammals or vocalization, tracks, presence of feces and other evidence such as carcasses. Surveys were carried out early in the morning (6-9 h), late in the afternoon (17-19 h) and during the night (20-23 h). Each month at least one week field activity was performed. Occasional traps were set to capture rodents and marsupials. All records were noted in a field form. An equal survey effort was carried out for each month during the whole field work.

To examine the effect of habitat utilization by the mammal species affected by shrinking and expansion of their habitats, we conducted an analysis of frequencies using the G-test (Sokal \& Rohlf, 1981). The G-test has been applied to biological research as it has theoretical advantages over the traditional $\chi^{2}$.

\section{RESULTS}

A total of 36 mammal species and 1,299 observations of individuals were recorded in the field. The most frequently observed species were the capybara Hydrochaeris hydrochaeris, the marsh-deer Blastocerus dichotomus, the fox Cerdocyon thous and the brocket Mazama gouazoubira. Table 1 summarizes the species and 


\begin{tabular}{|c|c|c|c|c|c|c|c|c|c|c|c|c|c|c|c|c|c|c|c|c|c|c|c|c|c|c|c|}
\hline & हैं & 의 & $\vec{n}$ & & -1 & 8 & - & -1 & $=$ & 인 & $\nabla$ & $\approx$ & 1 & & $\bar{\infty}$ & $\simeq=$ & $\simeq$ & $=0$ & N & 이 & & ה & $N$ & ஓి & $\nabla$ & -1 & $\stackrel{\infty}{m}$ \\
\hline & $b^{\circ}$ & 으 & ' & & & $\stackrel{\stackrel{\sim}{J}}{\sim}$ & & & iे & గె: & $\approx$ & $\nabla$ & & & $\stackrel{\vartheta}{+}$ & $\begin{array}{cc}m & \vdots \\
\infty & 0\end{array}$ & $\begin{array}{c}m \\
\infty \\
\infty\end{array}$ & $\vec{a}$ & & $\hat{\infty}$ & 14 & $\stackrel{\sim}{+} \stackrel{\sim}{+}$ & 요 & $\stackrel{m}{m}$ & ' & & $\exists$ \\
\hline & 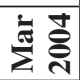 & & ' & ' & & $\underline{1}$ & & ' & - & - & - & -1 & I & ' & $\nabla$ & -1 & - & -1 & & $\nabla$ & 1 & $-1-$ & - & -1 & ' & & $\infty$ \\
\hline & $\Delta^{\circ}$ & 의 & $\stackrel{\infty}{a}$ & ' & ' & 弪 & & I & $\dot{\hat{n}}$ & ' & 1 & $\infty$ & I & ' & $\stackrel{⿱}{\sim}$ & $\begin{array}{c}m \\
\infty\end{array}$ & 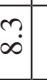 & & & & 1 & 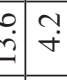 & ' & $m$ & $\approx$ & & $\stackrel{9}{r}$ \\
\hline & ¿ & - & $n$ & ' & ' & 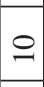 & & 1 & -1 & ' & . & $N$ & I & 1 & 0 & $-1-$ & - & & 10 & $m$ & 10 & $m-1$ & ' & - & -1 & & $m$ \\
\hline & $\Delta^{0}$ & 이 & ले. & ' & ' & $\overrightarrow{\mathrm{I}}$ & & 1 & ڤे. & $\begin{array}{l}\infty \\
\infty \\
\infty \\
-\end{array}$ & ' & 0 & ' & & $\stackrel{n}{n}$ & $\stackrel{m}{\infty}$ & 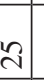 & $\vec{a}$ & & $m$ & 10 & 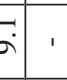 & ' & in & ' & & $\stackrel{n}{\varrho}$ \\
\hline & 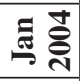 & - & $\sim$ & ' & I & $\infty$ & & ' & - & $m$ & , & $\nabla$ & , & 1 & $N$ & -0 & $m$ & -1 & . & 6 & . & $\begin{array}{lll}N & 1\end{array}$ & . & $a$ & ' & & $\nabla$ \\
\hline & $\Delta^{0}$ & & $\stackrel{\infty}{\sim}$ & ' & & \begin{tabular}{|l|} 
\\
\\
\end{tabular} & ' &. & \begin{tabular}{|c|}
$\hat{n}$ \\
\end{tabular} & חृ: & 1 & $\stackrel{\sim}{\sim}$ & I & ' & 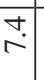 & . & 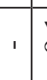 & $\vec{a}$ & l & $\vec{\infty}$ &. & 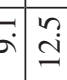 & ' & 의 & ' & & in \\
\hline & 苋 气ิ̊ & & $\nabla$ & ' & & $n$ & & ' & -1 & - & , & $r$ & ' & 1 & 0 & ' & & -1 & & $\nabla$ & o & $v m$ & . & $m$ & ' & & $N$ \\
\hline & $\Delta^{0}$ & 이 & in & ' & & $\overline{6}$ & & ' & $\mid \begin{array}{l}n \\
\text { nె. } \\
\end{array}$ & $\approx$ & 1 & $\simeq$ & ' & ' & $\stackrel{?}{0}$ & $\stackrel{0}{6}:$ & $\stackrel{-}{\underline{0}}$ & ' & 5 & $\stackrel{m}{\forall}$ & $1 \overline{0}$ & $\underset{\infty}{\infty}$ & 1 & $\stackrel{m}{m}$ & ' & & $\stackrel{9}{r}$ \\
\hline & zôे & -1 & $\infty$ & ' & & $\nabla$ & & 1 & $\nabla$ & $\nabla$ & 1 & $m$ & . & 1 & in & $N O$ & $N$ & & & $N$ & 10 & $N \mid N$ & . & $\nabla$ & ' & & $m$ \\
\hline & $b^{\circ}$ & & $\begin{array}{l}\infty \\
\infty \\
\end{array}$ & ' & ' & \begin{tabular}{|l|}
$\stackrel{0}{0}$ \\
\end{tabular} & & 1 & 1 & ' & in & $\infty$ & ' & 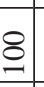 & $\begin{array}{l} \\
\dot{0} \\
\dot{2}\end{array}$ & $\begin{array}{l}m \\
\infty \\
\infty\end{array}$ & ' & 1 & & $\vec{a}$ & 17 & $\begin{array}{r}\sigma: 0 \\
\sigma\end{array}$ & ' & ㄱ. & $\approx$ & & m? \\
\hline & 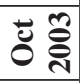 & & in & , & & $r$ & & , & 1 & , & $N$ & $N$ & I & - & $=$ & - & & , & & in & .0 & $v \mid \nabla$ & 1 & 0 & - & & 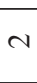 \\
\hline & $\Delta^{\circ}$ & 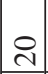 & $\stackrel{\infty}{=}$ & ' & & $\mid \begin{array}{l}0 \\
\stackrel{r}{r}\end{array}$ & & 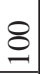 & $\begin{array}{l}a \\
\dot{n}\end{array}$ & भु. & 1 & $\nabla$ & ' & ' & $\vec{\imath}$ & & & & in & ?. & & 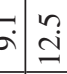 & 1 & $\hat{6}$ & ' & & m. \\
\hline & ڤัँ & $N$ & 0 & ' & ' & in & & - & - & - & . & -1 & I & ' & $\Xi$ & 1 & ' & $m-$ & - & $m$ & ' & $v m$ & . & $\mathrm{N}$ & ' & & $N$ \\
\hline & $8^{\circ}$ & 이 & 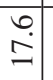 & ' & & $\stackrel{\vartheta}{*}$ & 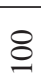 & ' & ì & ?̧: & $\approx$ & $\nabla$ & ' & ' & 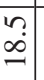 & 10 & $\begin{array}{c}n \\
\infty\end{array}$ & $\underset{\infty}{\infty}$ & in & $\stackrel{m}{+}$ & & 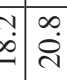 & 1 & $m$ & 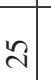 & & $\stackrel{2}{r}$ \\
\hline & $\stackrel{500}{\Xi}$ & -1 & $a$ & , & & $m$ & - & , & -1 & - & - & -1 & I & & $\cong$ & . & - & $N$ & -10 & $N$ & , & $+i n$ & . & -1 & - & & $m$ \\
\hline & $\approx$ & 의 & $\stackrel{\infty}{\sim}$ & ' & & $m$ & & 1 & \begin{tabular}{|l}
$\vec{j}$ \\
$\dot{n}$
\end{tabular} & mु. & ' & & I & ' & $\stackrel{+}{\sim}$ & 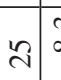 & $\begin{array}{l}m \\
\infty \\
\infty\end{array}$ & $\bar{a}$ & ' & $m$ & $\bar{c}$ & $\vec{\sigma}: \hat{\sigma}$ & in & $\hat{6}$ & $\approx$ & 8 & $\stackrel{9}{r}$ \\
\hline & 至 & - & $\nabla$ & ' & & $N$ & 1 & I & -1 & - & , & & ' & ' & 0 & $m-$ & - & -1 & ' & 6 & c & $N \mid \nabla$ & - & $\sim$ & -1 & -10 & $m$ \\
\hline & $x^{2}$ & & aे. & ' & 8 & $\stackrel{n}{-}$ & & 1 & $\begin{array}{l}a \\
\dot{n}\end{array}$ & $n$ & 1 & $\nabla$ & I & ' & 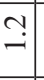 & ' & & $\vec{a}$ & ' & $\vec{\infty}$ & 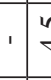 & \begin{tabular}{l|l}
$\stackrel{f}{f}$ & $\stackrel{f}{*}$
\end{tabular} & , & & I & & ' \\
\hline & 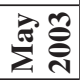 & & $m$ & ' & - & - & & , & -1 & $N$ & . & - & , & I & -1 & , & & - & & $\checkmark$ & - & -- & ' & & ' & & ' \\
\hline & $\Delta^{\circ}$ & 이 & $\begin{array}{l}\infty \\
\infty \\
\infty\end{array}$ & & & r & & ' & $\begin{array}{l}+ \\
\ddot{\lambda}\end{array}$ & $\begin{array}{l}n \\
\text { I } \\
\end{array}$ & ' & $\simeq$ & ' & & $\sigma^{2}$ & $\approx$ & 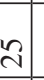 & $\bar{a}$ & & $\begin{array}{l}n \\
n \\
n\end{array}$ & 5 & $\stackrel{?}{+}$ & ' & $\stackrel{m}{m}$ & ' & & $\vec{\Delta}$ \\
\hline & $\dot{\bar{z}}$ & & $n$ & ' & & in & & ' & $n$ & $N$ & 1 & $m$ & , & & $\infty$ & $m$ & $m$ & -1 & & 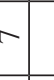 & ' & ' & . & - & ' & & $\infty$ \\
\hline & 莺 & 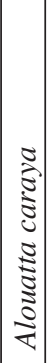 & 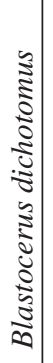 & 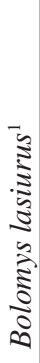 & 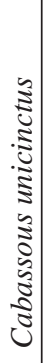 & \begin{tabular}{|c} 
\\
0 \\
0 \\
0 \\
0 \\
0 \\
0 \\
0 \\
0
\end{tabular} & 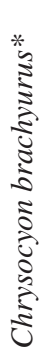 & 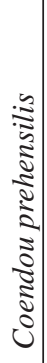 & 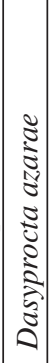 & 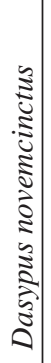 & $\begin{array}{c} \\
0 \\
5 \\
0 \\
0 \\
5 \\
0 \\
0 \\
0=1\end{array}$ & 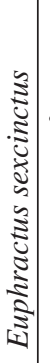 & 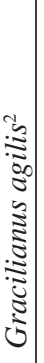 & 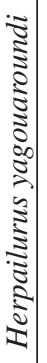 & 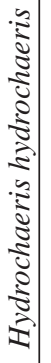 & 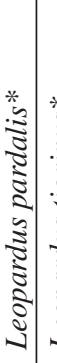 & 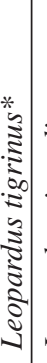 & 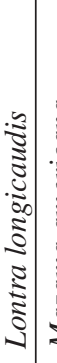 & 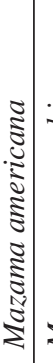 & 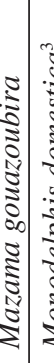 & 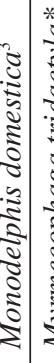 & 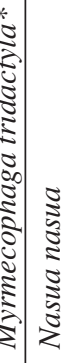 & $\mid \begin{array}{c}* \\
0 \\
0 \\
0 \\
0 \\
0 \\
0 \\
\tilde{0} \\
\tilde{0} \\
0 \\
0 \\
0\end{array}$ & 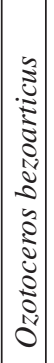 & 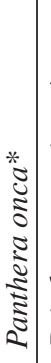 & 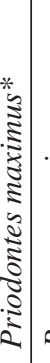 & 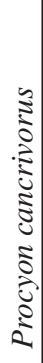 \\
\hline
\end{tabular}




\begin{tabular}{|c|c|c|c|c|c|c|c|c|c|c|c|}
\hline \pm & ণิ & ' & $r$ & $=$ & ল & & $=$ & 0 & $=$ & ஜ & \\
\hline ڤ. & 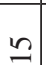 & ' & $\begin{array}{l}\dot{0} \\
\dot{d} \\
\dot{d}\end{array}$ & ' & $\infty$ & & i. & 1 & $\begin{array}{l}1 \\
\infty \\
\infty\end{array}$ & $\tilde{a}$ & on $\frac{0}{0}$ \\
\hline-1 & $m$ & ' & $N$ & ' & ल & & -1 & I & $N$ & $\stackrel{\bullet}{n}$ & \\
\hline aे & $n$ & ' & ' & ' & $\infty$ & & \begin{tabular}{l|l}
$v$ & 0 \\
$\infty$ & 0 \\
0 & 0
\end{tabular} & $\begin{array}{l}\infty \\
\infty \\
\infty\end{array}$ & $\stackrel{1}{\infty}$ & $\begin{array}{l}0 \\
\infty\end{array}$ & 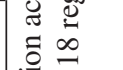 \\
\hline-1 & -1 & & & ' & $\forall$ & & $v a$ & $m$ & $\mathrm{~N}$ & in & $\stackrel{0}{0}$ \\
\hline $\begin{array}{l}a \\
n\end{array}$ & $\approx$ & I & $\stackrel{\leftrightarrow}{\dot{\gamma}}$ & $\vec{a}$ & 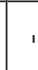 & & ' & $\stackrel{n}{\simeq}$ & $\vec{a}$ & $\hat{a}$ & 壳 \\
\hline-1 & $n$ & ' & $m$ & - & ' & & ' & $\mathrm{N}$ & - & i & 류유 \\
\hline 1 & 1 & ' & ' & $\stackrel{N}{\infty}$ & o & & 1 & mु? & $\vec{a}$ & $\stackrel{+}{r}$ & 氖 \\
\hline I & 1 & ' & I & $N$ & 0 & & ' & -1 & - & 年 & . \\
\hline$\infty$ & 1 & 1 & ' & $\vec{a}$ & $\infty$ & & ' & $\begin{array}{l}\infty \\
\infty \\
\infty\end{array}$ & $\vec{a}$ & ナ. & 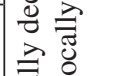 \\
\hline$\sim$ & 1 & I & ' & - & $\nabla$ & & ' & $m$ & - & in & $\stackrel{\breve{E}}{\mathscr{E}}$ \\
\hline 1 & 1 & I & ' & ' & $\infty$ & & $\bar{a} \cdot \mathrm{s}$ & ?ु! & $\vec{a}$ & $\bar{a}$ & $\cdot \frac{\infty}{8}$ \\
\hline 1 & 1 & ' & ' & ' & ल & & -1. & -1 & - & $n$ & $\begin{array}{l}\text { की } \\
*\end{array}$ \\
\hline 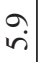 & $n$ & ' & ' & ' & 2 & & $\overline{0}$ & m? & $\vec{a}$ & $\ddot{n}$ & \\
\hline-1 & -1 & ' & ' & ' & - & & -1. & -1 & - & in & $\stackrel{0}{0}$ \\
\hline ñ & 의 & ' & ' & $\stackrel{?}{\pi}$ & in & & 1 & ?ु? & $\vec{a}$ & $\ddot{0}$ & $\stackrel{\Xi}{\Xi}$ \\
\hline$\nabla$ & $N$ & ' & I & $m$ & 0 & & ' & -1 & - & ర & 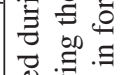 \\
\hline $\begin{array}{l}0 \\
\vdots \\
-1\end{array}$ & 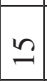 & ' & $\begin{array}{l}\infty \\
\infty \\
\infty\end{array}$ & $\begin{array}{l}N \\
\infty \\
\infty\end{array}$ & I & & 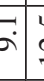 & ¿n & . & $\begin{array}{l}0 \\
\dot{0}\end{array}$ & 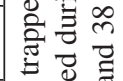 \\
\hline$m$ & $m$ & ' & $N$ & $N$ & in & & -10 & $N$ & 1 & $\stackrel{\infty}{n}$ & 胥 \\
\hline aे & 1 & I & ' & ' & $\stackrel{2}{r}$ & & ? & 1 & 1 & $\stackrel{\circ}{\dot{m}}$ & $\begin{array}{l}\text { Еี } \\
\mathbb{ల}\end{array}$ \\
\hline-1 & 1 & I & ' & ' & - & & $m$ & 1 & 1 & 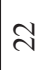 & 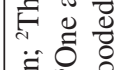 \\
\hline $\begin{array}{l}0 \\
\vdots \\
-1\end{array}$ & $\approx$ & I & ' & $\stackrel{N}{\infty}$ & r & & 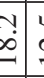 & $\stackrel{n}{\mathfrak{c}}$ & $a$ & 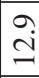 & 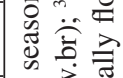 \\
\hline$m$ & $n$ & I & I & $N$ & 0 & & $\mathrm{~N}$ & $N$ & - & $\infty$ & ठే \\
\hline 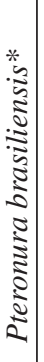 & $\mid \begin{array}{c} \\
\\
* \\
\vdots \\
\vdots \\
0 \\
0 \\
\vdots \\
0 \\
0 \\
\vdots \\
\vdots \\
\vdots \\
0 \\
0\end{array}$ & $\frac{0}{0}$ & 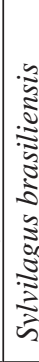 & 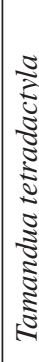 & 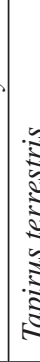 & 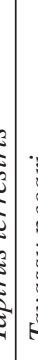 & 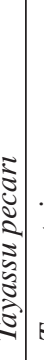 & 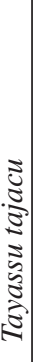 & 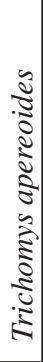 & 苂 & 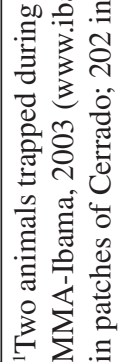 \\
\hline
\end{tabular}


their frequency of observations throughout the year. Aquatic species such as the giant river otter Pteronura brasiliensis and the freshwater otter Lontra longicaudis were observed, of course, only in the transect along the river.

There is observed evidence that seasonal habitat availability of the Pantanal influences population parameters of mammal species (Table 1). By comparing observed frequencies of animals in the transects, a frequency test ( $\mathrm{G}$ test) applied to check the difference of habitat utilization by the species showed statistically significant differences among the observed habitats - seasonally flooded grassland, patches of Cerrado, gallery forest and mesophytic forest - (G-value $=191.5$; with 60 degree of freedom; $\mathrm{n}=605 ; \mathrm{P}<0.001)$ and the seasonally flooded grassland was the most utilized habitat by the species (31\%), followed by the mesophytic forest $(29.6 \%)$ and gallery forest $(15 \%)$. Thus, there is a significant association of species utilization and kind of available seasonal habitat.

The months with a greater number of observations of individuals were August and September, coinciding with the dry season, when more expansion of grassland fields (seasonally flooded) is observed. Fig. 1 shows the results for all species during the study year.

\section{DISCUSSION}

The fauna of the Pantanal has a strong biogeographical influence of the surrounding Cerrado (Alho et al., 2003; Alho \& Gonçalves, 2005). It is well known that while the diversity of species, the Cerrado, is greater than the diversity in the Pantanal, on the other hand the same species which have larger geographical distribution find exuberant seasonal abundance in the Pantanal due to the offer of annual reproductive and feeding niches. This is the case of opportunistic species

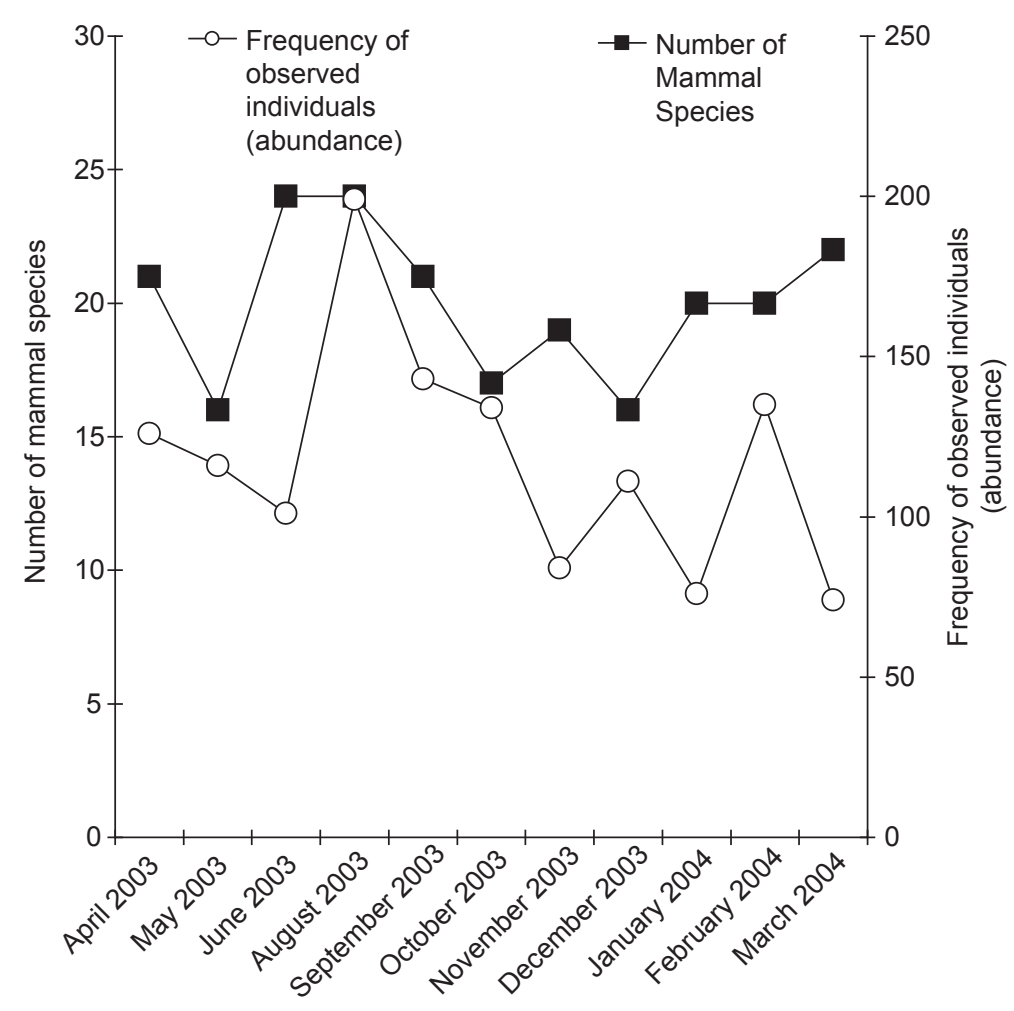

Fig. 1 - The frequency of observed individuals (abundance) is higher during the dry season, when the land dries out and an expansion of terrestrial habitats occur, while the number of observed mammal species remain stable throughout the year. 
that take advantage of the seasonal shrinking-andexpansion of habitats to move into new habitats when the land dries out, making movements among forested and open seasonal habitats or by increasing the number through reproductive efforts to occupy the available space. This is the case of the capybara Hydrochaeris hydrochaeris, the marsh-deer Blastocerus dichotomus, the coati Nasua nasua and the crab-eating-fox Cerdocyon thous. Small mammals such as wild rodents and marsupials apparently have a strong influence on annual population parameters, due to the shrinkingand-expansion of habitats. Terrestrial rodents like Trichomys apereoides, relatively abundant in the Pantanal, frequently occur in habitats of savanna outcroppings in the Cerrado biome, but are found in patches of forest, near seasonally flooded areas of the Pantanal, with no rocks (Lacher et al., 1986; Alho et al., 2003). In the Pantanal, the higher ground (where there are patches of savanna and "cordilheira" of woodland) is only a couple of centimeters above the average water level. Studies on small mammals in the Pantanal and Cerrado biomes (Lacher et al., 1986; Lacher \& Alho, 1989; Mares \& Ernest, 1995) show that different species have distinctive patterns of population and reproductive activity, with fluctuations between the dry and wet seasons, depending on the availability of ecological resources (food, reproductive niche and space).

Various case studies carried out throughout the Pantanal support the relationship between flooding regime and life cycles of plants and animals (Alho, 2005; Alho \& Gonçalves, 2005). Fish cycle, for example, is strongly related to the water regime in the Pantanal (PRODEAGRO, 1997). Most waterfowl species show synchronized reproduction, where huge colonies of birds such as wood storks, egrets and spoonbills concentrate in nesting sites in the gallery forest during the dry season to take advantage of the offer of fish and other animals "trapped" in small pools during the dry season (Alho \& Gonçalves, 2005). Studies on capybaras show that the use of habitats varies seasonally (Alho et al., 1989). During the dry season, capybaras spend the night in the forest. In the early morning they leave the forest to graze on the grassland. During the rainy season, the capybaras also spend the night in the forest, but in the morning usually emerge and go directly to the water or to grazing areas. Capybaras use aquatic and forest vegetation in their diet and, at this time, it increases significantly, as few grazing areas remain above the water level.

All vegetation communities in which marsh deer have been observed (Tomás, 1993) are frequently flooded during the wet season (most habitats are formed by aquatic plants). Seasonal flooding causes significant variations in the population distribution and density. During the dry season, deer prefer the boundary between the flooded areas and the drained range of the marshland. During the flood season the animals are dispersed. The Pantanal jaguar, for example, can swim very well and is highly adapted to the flooding pattern.

This study emphasizes the importance of the hydrological flow of water from the surrounding plateaus to the flooding plains, causing the seasonal shrinking and expansion of available habitats of mammal species in the Pantanal. Mammal species seem to be well adapted to these annual changes by exploring open and forested habitat. Thus, forested habitats such as gallery forests, mesophitic forests (such as Cerradão) and arboreal habitats like patches of cerrado are crucial for mammalian species in the Pantanal. Even arboreal species such as the primate howler monkey Alouatta caraya, essentially a mixed feeder relying on new sprouts of trees, flowers and fruits, depend on the flooding regime of the Pantanal, which provides the phenological rhythms for the offer of food supply. The Pantanal is part of the national heritage of Brazil and the knowledge of its exuberant fauna is fundamental for the conservation and rational use of the biome.

Acknowledgments - This study was supported by a grant from the Fundação Manoel de Barros from the UNIDERP Universidade para o Desenvolvimento do Estado e da Região do Pantanal, Pós-graduação em Meio Ambiente e Desenvolvimento Regional, sub-project on terrestrial mammals in the Pantanal.

\section{REFERENCES}

ALHO, C. J. R., 2005. The Pantanal, pp. 203-271. In: The World's Largest Wetlands - Ecology and Conservation, Edited by Lauchlan H. Fraser \& Paul A. Keddy, Cambridge University Press, N.Y.

ALHO, C. J. R. \& GONÇALVES. H. C., 2005, Biodiversidade do Pantanal - Ecologia \& Conservação, Editora UNIDERP, Campo Grande, 135p. 
ALHO, C. J. R; LACHER JR., E. T. \& GONÇALVES, H. C., 1988, Environmental Degradation in the Pantanal Ecosystem. BioScience, 38: 164-171.

ALHO, C. J. R. \& GONÇALVES, H. C., 2004, The Pantanal wetland of Brazil: the global importance of its biodiversity, ecological processes, and analysis of threats for conservation. Abstract (Page 11), 7th INTECOL. International Wetlands Conference; Utrecht, Holand.

ALHO, C. J. R., STRÜSSMANN, C., VOLPE, M., SONODA, F., MARQUES, A. A. B., SCHNEIDER, M., SANTOS JÚNIOR, T. S. \& MARQUES, S. R., 2003, Conservação da Biodiversidade da Bacia do Alto Paraguai, Editora UNIDERP, Campo Grande-MS, 466p.

ALHO, C. J. R., CAMPOS, Z. M. S. \& GONÇALVES, H. C., 1989, Ecology, Social Behavior, and Management of the Capybara (Hydrochaeris hydrochaeris) in the Pantanal of Brazil, pp. 163-194. In: Advances in Neotropical Mammalogy, edited by Kent H. Redford and John F. Eisenberg. University of Florida, Gainesville, Sandhill Crane Press.

ANA, 2004, Agência Nacional de Águas (National Water Agency of Brazil). Programa de Ações Estratégicas para o Gerenciamento Integrado do Pantanal e Bacia do Alto Paraguai. Website of the GEF Pantanl/Upper Paraguay Project http://www.ana.govbr/gefap/

FERNANDES, E., LUCATI, H. L. CAPELLARI, B. C. \& QUEIROZ NETO, J. P., 1996, Modelo digital para tratamento de cartas topográficas do Pantanal da Nhecolândia, pp. 159-166. In: Anais do II Simpósio sobre recursos naturais e sócio-econômicos do Pantanal, CPAPEMBRAPA e UFMS, Corumbá, MS

FRANCO, M. S. M. \& PINHEIRO, R., 1982, Geomorfologia, pp. 161-224. In: Brasil, Ministério das Minas e Energia. Departamento Nacional de Produção Mineral. Projeto RADAMBRASIL. Folha SE. 21 Corumbá and SE 20. Levantamento de Recursos Naturais, 27. Rio de Janeiro.

HAMILTON, S. K., SIPPEL, S. J. \& MELACK, J. M., 1996, Inundation patterns in the Pantanal wetland of South America determined from passive microwave remote sensing. Arch. Hydrobiol., 137: 1-23.

JUNK, W. J., 1993, Wetlands of tropical South America, pp. 679-739. In: D. Whigham et al. (eds.), Wetlands of the World: inventory, ecology, and management. V.1 Kluwer.
LACHER, Jr., T. E. \& ALHO, C. J. R., 1989, Microhabitat Use Among Small Mammals in the Brazilian Pantanal. $J$. Mamm., 70(2): 396-401.

LACHER, Jr., ALHO, C. J. R. \& CAMPOS, Z. M. S., 1986, Densidades y Preferencias de Microhábitats de los Mamíferos en la Hacienda Nhumirim, Sub-Región Nhecolândia, Pantanal de Mato Grosso del Sur. Ciencia Interamericana, OEA, Washington, 26(1-2): 30-38.

MARES, M. A. \& ERNEST, K. A., 1995, Population and community ecology of small mammals in gallery forest of Central Brazil. Journal of Mammalogy, 76(3): 750-768.

PCBAP, 1997. Plano de Conservação da Bacia do Alto ParaguaiPCBAP, v.II, t.III. Diagnóstico dos meios fisicos e biótico, meio biótico. Brasília: MMA/PNMA, 1997. 433p.

PRODEAGRO, 1997, Projeto de Desenvolvimento Agroambiental do Estado de Mato Grosso. Governo do Estado de Mato Grosso. Cuiabá. Paper in digital format (CD). Meio Biótico

REDFORD, K. H. \& EISENBERG, J. F., 1992, Mammals of the Neotropics. The University of Chicago Press, Chicago, USA.

SOKAL, R. R. \& ROHLF, R. J., 1981, Biometry: the principles and practice of statistics in biological research. Second ed. W.H. Freeman and Company, San Francisco, California, USA.

TNC (The Nature Conservancy), 2003, Classificação dos Ecossistemas Aquáticos do Pantanal e da Bacia do Alto Paraguai. Brasília, 108p.

TOMÁS, W., 1993, Status and Ecology of a Marsh Deer (Blastocerus dichotomus) population in southern Pantanal, Brazil. Unpublished Report. WWF. Brasília.

VINSON, M. R. \& HAWKINS, C. P., 1998, Biodiversity of stream insects: variation at local, basin and regional scales. Annual Review of Entomology, 43: 271-293.

WANTZEN, K. M. \& JUNK, W. J., 2000, The importance of stream-wetland-systems for biodiversity: a tropical perspective. In: Gopal, B.; Junk, W. J.; Davis, J. A. (eds.) Biodiversity in wetlands: assessment, function and conservation. Leiden: Backhuys Publishers, 1: 11-34. 